\title{
Research of Optimal Path Automatic Navigation in the Prescribed Scope
}

\author{
Zou $\mathrm{Ji}^{1}$, Zheng $\mathrm{Jie}^{1}$ and Bai Duanyuan ${ }^{2}$ \\ 1Electronic and Information Engineering College, Changchun University \\ Changchun City, Jilin Province, China \\ 2Electronic and Information Engineering College, Changchun University of \\ Science and Technology \\ Changchun City, Jilin Province, China \\ 13517589@qq.com,384539844@qq.com,11267243@qq.com
}

\begin{abstract}
Identification, liquidity and the direction of the blind and weak-sight pat ents are more and more hard, and strange scenario especially. Introduces the embedded pedestrian navigation system consists of sensor, they are active radio frequency. Identification (radio frequency identification devices) tags active-system System navigation formed in the active radio frequency identification is a kind of detecting direction way, determine the any positions and directions of weak-sight through intelligent network labels. The direction of the experimental data proved that forany navigation positioning and distance are effective in the range of intelligent network
\end{abstract}

Keywords: Optimal path; Scope of navigation: Radio frequency identification tag; Route simulation planning

\section{Introduction of System Contents}

Positioning and navigation services has been attention with the rapid development of science and technology more and more. As we all know, the GPS (global positioning system) equipment are directions and navigating services. Global positioning system has been widely used into any ways. Positioning and navigating function compound of scenes or area, especially in a closed scope positioning and navigation is important. A large special place navigation, increasing trade prospects in the near future.

User must constanty with satellite based on GPS system, GPS system, it is one of the biggest limit, this is the line of sight environment. The position precision cannot reach the required standad, if the system work, error due to the Rf signal coupling transmitting is restricted into a scope of limited and closed within a building. GPS accuracy of about 6 meters, the highest accuracy cannot meet the requirement of the metre indoor navigation. Anyone need the function simply speech positioning and navigation system, in some outdoor and indoor place, such as shops, parks, playgrounds, art museum, square, etc. But they can't meet the needs of the blind complicated navigating area.

Users for example children or sight-weak people, they must rely on a lot of signals display direction and direction. A innovation regional navigating equipment comes from radio frequency identification and voice way to meet a requirements of indoor navigating complex region.

In fact, a RFID-tag equipment will not tell others which way to forward without voice positioning and navigation system. Once the need to improve the accuracy, and the RFID application integration of speech recognition technology is an extremely important in the area navigation within the scope of application, and have the function of positioning. As of this year, the function of route guidance device has been used in most of the developed countries. Set up by a sound good sensors placed in items hidden place, five separate 
transmission interference voice messages. Once the user foot proximity sensors, users through carrying receiver for broadcast exhibitors the voice exhibition to introduce information of language acquisition in advance. In this way the user can create a kind of brand-new information voice device, through the use of the radio frequency tag identification methods and the combination of information language provide navigation services.

At the same time, many nonprofit organizations and large service companies need study RFID for large services for a fee. Here will design a complete system of navigation and positioning, voice equipment used in information services and the embedded application of portable mobile devices.

This purpose a grid of information for the design of RFID tag path should be for the blind or visual defect provides a safe navigation, and form a large comprehensive network systems and related voice signal database established, think that people in the navigation and positioning of the route remind him when readers out of safety grid region. In the actual environment, when using the blind and visually handicapped by rf signal under the condition of coupled to the electromagnetic wave, the system can also be output according to the address of the database to a synthetic artificial speech, thus provides the user with a clear audible voice services.

\section{System Navigation Construction}

System the application of RFID tags, compact device as a navigation receiver and plan route of travel or when walking in a particular area.

The system will be loading the codes roicing navigating signals. A ground node identification tag will be installed. Code speech or installation position need change comes from the use of network.

If binding or sight-weak using a routing node installation identification tag, RFID reader's embedded portable handheld navigating devices to get signal with the net-tags, remind any blind route tactile instructions or a speaker. Players will use the blue - teeth combined with hand-held devices. As binding men and sight-weak need get to the scope location navigating after vice information. Development platform

System will HP IPAQ h2100 PDA, intelligent terminal some successful projects in China. Platform sortware studio Visual C language based on mobile devices in 2007 and wince operating system. Database are built system. Considering the computing power of mobile devices and the cost the data will be as an XML file, rather than the database file is retained in the local nobile device.

The development of ymportant technology in the equipment.

Three system important technology, RFID localization, path planning, voice services..

\subsection{RFID Tag and Writer Introduction}

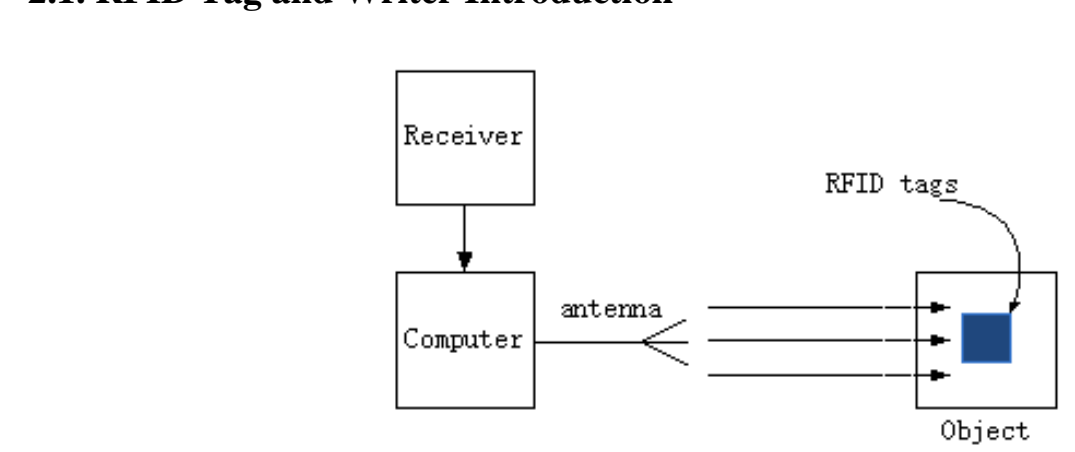

Figure 1. Radio Frequency Identification Tags Net Key System Block 
Electric coupling signal frequency identify is not a kind of advanced methods. It is a signal coupling network methods, RF signal access to coupling data to the labels. A working principle of equipment into all kinds of scope ways, that recognition getting.

This basic foundation based on radio frequency identification methods are routed. Will trigger the user information get to the labels within scope, tag for magnetic radiation, passive tags will be induced current, the information in the memory chip and word tags must emit the coupling information. Information need to transfer to of information processing system if readers access to return information and decoding

\subsection{Route Simulation Optimal Planning}

Network ALG comes from close loops and open loops. Network no excitement, and exciting node will be an end of the timetable.

A) First of all, the initial point of the nearest point without checking will row Open the node group, and then check.

B) Found that all of the branch node nearest starting point, and will in the end of the timetable.

C) Research branch node, initial point distance computing nodes, brânch node in the networks.

D) Repeating steps two and three until the turn on and clear up or arrange target.

System path algorithm to achieve the optimized way classical Diijkstra ALG, is used to calculate a way hot to get to point. The shortest path AL G need calculate methods ways.

Speech identify.

A purpose of the speech services into spech recognitionsystem, the directing and positing of actual-timing voice reminder. The directing or positing of the speech remind actual-timing application of recode speech comes from the database. Important inputting is usually the preferred portable handed in PD important inputting doesn't fit in the processing of moving. The important is methods to users via voice command to select a destination. Therefore, one of the key technologies of automatic speech recognition equipment.

As practice, obtain ormake use of the other characteristic parameters as inputting language. Parameters vill complex in the tag, if been training in good, language way and dictionary indexing decoding. Finaliy, according in sound waves will language. Model of text model (HMM) and other necessary language model such as model (LM) into achieve the optimized way, the maintarget language is mandarin.

Key vocabulary target focused on field condition, such as indoor object. Navigating will be completed which the site access position.

No problem be olved many speech recognition algorithm, although the voice recognition method has should turn to practical use. Especially small and medium vocabulary voice recognition ALG is nearing completion, has been widely used with portable equipment. This is a reliable effective network construction also.

\section{Research of Range of Distance Navigation Algorithm}

Introduction of localization model and ALG

Completed network integrated RFID system can be modeled as a complete branches are connected directed parameter $\mathrm{G}=(\mathrm{V}, \mathrm{E}), \mathrm{V}$ is used to gather any nodes information scope, "E" is for all of the information collection part of the navigation route. Our collection () the information is known to all the nodes position. Direction collection of search position, every position of set must to get the under method:

$$
\begin{gathered}
0<L_{i-j 1} \leq L_{i-j 2} \leq \ldots \ldots \leq L_{i-j k} \leq R \\
L_{i-j x}(1 \leq x \leq k)
\end{gathered}
$$


On behalf of a special node point and point of relative distance, we position by setting all nodes information transmission path is the same type of signal. By using the voronoi diagram of inequality. Estimate the position error

Based on tyson form the use of multilateral significance of network, the user can set the arbitrary polygon method of area began to place. From the data points in the same direction, if the maintaining of elimination, you can know the rest of the information from the most recent data module to access the data node process, so this definition in the voronoi region, can repeat this process until all computing voronoi region of the anchor node input is completed, and then calculate tyson more complex cross hybrid areas shall be defined algorithm calculation point in the end.

\section{Structure Subsystem Objects Database Model}

System objects Scenarios on table of applicable

Table 1. Applicable Objects Scenarios

\begin{tabular}{|l|l|l|}
\hline \multicolumn{2}{|c|}{ Applicable scenarios objects dictionary } \\
\hline XML-version & Door & Pipe \\
\hline Location & Window & Tele ision \\
\hline Latitude & Porch & Couvuter \\
\hline Longitude & Road & Rhone \\
\hline Height & Floor & Retrigerato \\
\hline Path & Stair & Washer \\
\hline Corridor & Handrail & CD-player \\
\hline Gymnasium & Mirrior & Light \\
\hline Cinema & Desk & Pot \\
\hline Hospital & Bed & Tank \\
\hline Restaurant & Chair & Thermostat \\
\hline Toilet & cabm & Dicrowave \\
\hline Bathroom & Poor & hangers \\
\hline Kitchen & Stage & Dispenser \\
\hline Bedroom & Curb & Guideboard \\
\hline Faucet & Decelernting bett & Garbage can \\
\hline Corridor & Directindoard & Tree \\
\hline
\end{tabular}

Navigation devices are work to display pedestrian or the user through the route and $\mathrm{rf}$ reading articles or scenario, label on the space distribution in each position, thus bring great convenience of use in the conditions, and thus feasibility for the expansion of system.

The structure of the deve system includes two parts, with parts and network nodes.

Using computer aided design system of vector diagram is to facilitate the think the map information related to set and optimize the design of the route. Handheld device also, of course, is very important, so the user use it all the time.

\subsection{Personal Computer Application System}

Used to implement microcomputer system of map information can import some importan geographic information. Technology and will transform these important geographic information for label nodes, used to match a special local scope in the appopriate figure need gathering places or objects. All the important geographical indication, such as object location, name, physical objects, labels, and order, will result in a form of XML file is stored in the memory of the background. While reading tag data transmission equipment, only need to do is finished loading the background information of XML documents in handheld devices. 


\subsection{Handheld Personal Digital Assistant System}

Handheld devices is the basic function of geography and the object information, and the database with the electromagnetic coupling signal corresponding address query. Users through continuous moving route navigation and optimized recognition.

Orientation must be the judge in addition to the position, Handheld system can be calculated according to the next node, thus to determine the direction by determining the label order. As shown in Figure 7 says the process of order. When the transponder is placed at the edge of the grid for the first time the center of the face, RFID equipment (micro controller is responsible for the direct RFID reader tag information) from the user's information data number. This tag is to find information id tags. Response controller, and then retrieve the label information matching has good distribution table of the input, the determine the relevant tag will be located in the grid. The distribution of the database in advance label value symbol navigation route will be sent to the controller (microcontroller task execution navigation algorithm).

\subsection{Maps and Speech Patterns}

Hand-held devices will load map suitable information file tơ faciliate optimal route ahead of time. Has been used to edit related vector map files in the node location information will be used to load in advance in the system memory. On the normal use of portable devices, data map will be displayed on a big screen on the handheld device also shows the time and other data.

Navigation and positioning, including roufe planning be used in real time, and meet the response time of the system. Corresponding time not more than 2.5 seconds, enough for most people the map navigation requirements. Further improve the precision of the corresponding time for 1 second.

a) Rf -label optimization selection

According to international standard protocol, node data transfer rate is $64 \mathrm{Kbps}$ can satisfy the requirement of the system transmission speed. So the system only gives a rate of 64 distance is drawing tag ID is 8 bytes of data size our actual choice.

b) Tag measures of antivinterference way

A few years ago the European telecommunications standards institute has developed a based on RFID protocol standard for the European Union, this is a kind of multimodal ways of working, the working principle of this protocol is similar to CSMA enhancement technique. Before users can try to communicate with the tag, the transmission of information is free, and then the user can query the relevant set label information, but need to wait for a free channel.

There is no inferference between users and consumers, because each receive channel. But does not solve the coupling interference between two adjacent label.

c) Radiation frequency protocol standard

Active RFID system of labels and reading and writing are outward radiation power at work, but the output power is very small, generally less than $10 \mathrm{mw}$ (433 MHZ, 5.8 Gz). Active electronic tags did not enter the working state, does not produce electromagnetic radiation. And mobile phone radiation power (100 $\mathrm{mw} \sim 1 \mathrm{w})$, active RFID system completely belongs to the small signal radiation, won't cause any influence to the human body.

Normal adults, passive RFID read-write device in the UHF frequency band antenna radiation within the scope of the maximum power, maximum radiation direction of half a meter away from antenna distance, resident time less than 6 minutes, then on the basis of radiation limit rules (see Table $1 \sim 4$ ) standard, which can determine the body on the electromagnetic radiation completely within safe limits.

d) Interference elimination - writer group reader 
Once users using the server/client to access the database and SQL software for Sorve7.0 base data address. Every tag position need transmit information in this SQL database, if the network access and terminal nodes can be used as a reference.

\section{To Compare with Global Position System}

Compared with the GPS navigation and positioning system in the region have great advantage in the local scope.

Level of civilian GPS positioning accuracy is more than two meters distance, but our inheritance of navigation and positioning system can make the user's accuracy can be less than 0.5 meters. Even small small 1 objects can also be identified as route of obstacles, the new integrated system can be used for up and down on the floor or highly navigation is the vertical height. If RFID network combining GPS can increasing the use of more convenience.

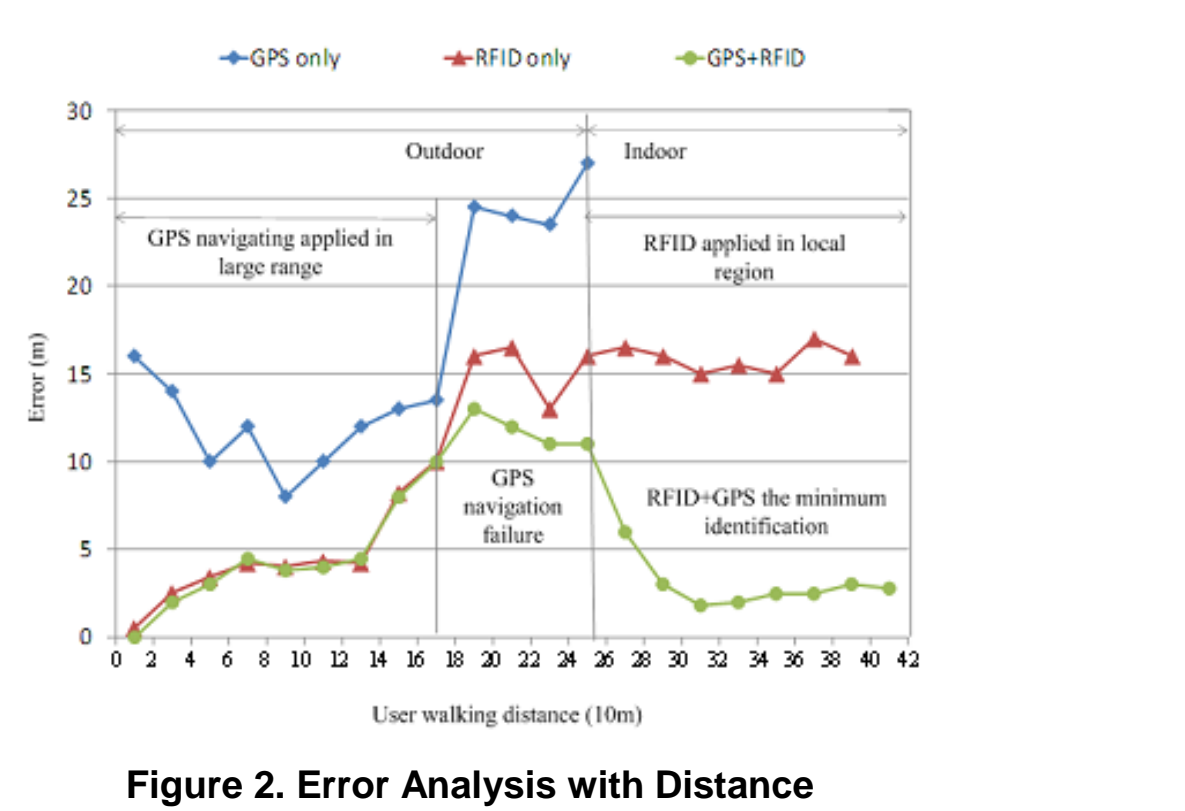

The second ard following pages should begin 1.0 inch $(2.54 \mathrm{~cm})$ from the top edge. On all pages, the bottom margin should be $1-3 / 16$ inches $(2.86 \mathrm{~cm})$ from the bottom edge of the page for $85 \times 11$-inch paper; for A4 paper, approximately $1-5 / 8$ inches $(4.13 \mathrm{~cm})$ from the bottom edge or the page.

\section{The Optimal Path Planning}

The portable terminal and mobile devices can be pre-loaded with relevant decision analysis model, so that you can appear in the center of the scene or may, according to situation, automatically provides a recommended solution, for staff reference, determine the scientific and reasonable decision or plan. The control center can know the location of mobile terminals and status information in time, thus to effective tracking of assets, in order to ensure safety; to reasonable allocate resources, in order to ensure the efficiency of production.

Based on the current mobile terminal location and the location of the destination, the optimum route analysis, and according to the preset conditions and rules, generated advice route to destination, at the same time provide crucial information along the way.

The comprehensive application of GPS and GIS platform, through the use of mobile communications, the Internet and spatial information technology and means, which is based on electronic map, on the basis of the location of the mobile terminal data, let users 
can get all kinds of information related to the current position, thus to work provides the reference for the further development of the basis.

Through ArcGis plug-in and TatukGis calculated after get the fastest path:

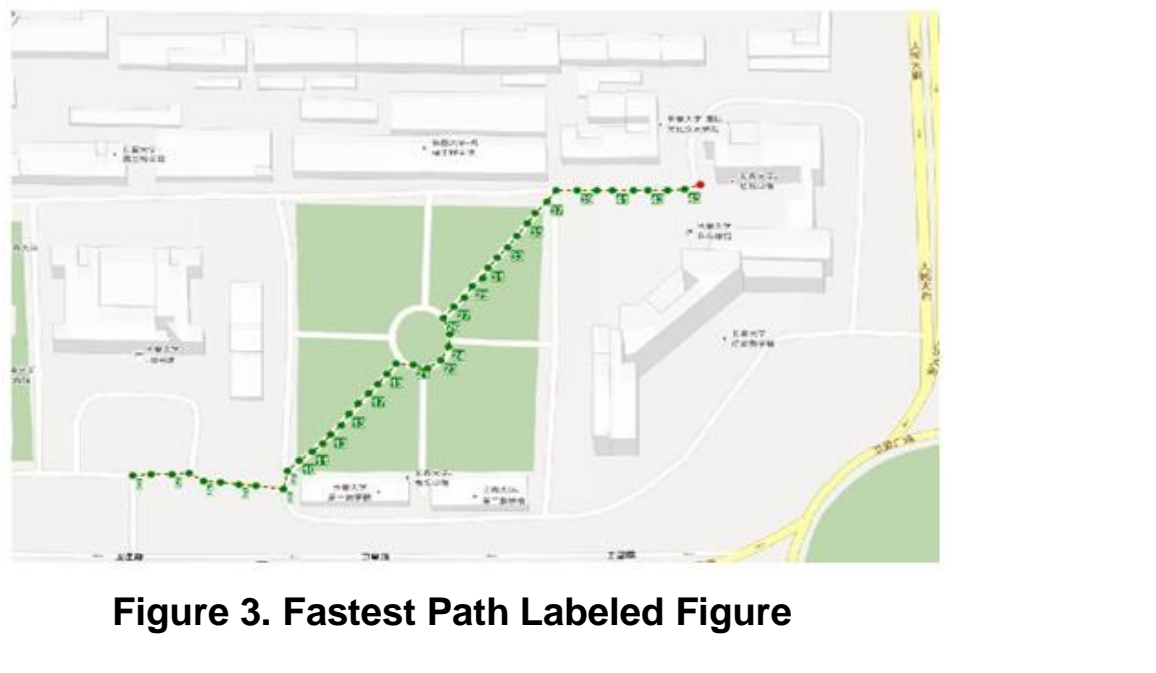

After the route of the contrast optimization, shown in the figure below:

This solution is to use GPS navigation as the main equipment the future can expand to beidou navigation system, in line with the system multiplexing, improves the stability and accuracy of the system.

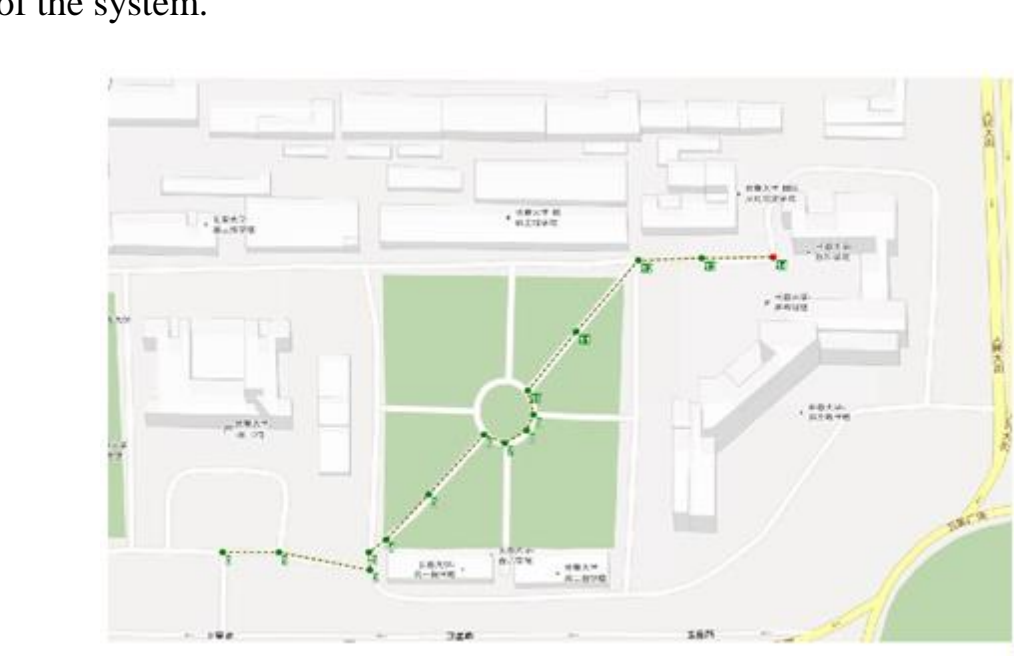

Figure 4. The Optimal Route Simplified Diagram

\section{Conclusion}

This paper tell a case based on RFID enhancement technique used for navigation, including the design of the map, route optimization algorithm, the design of handheld portable, speaking, reading and writing as well as phonetic database of supervision and so on.

The new system not only provide users with free travel convenience, more people vision defects provided voice navigation needs, thus extending the interpretation of some of the large range of buildings or exhibition. 


\section{References}

[1] L. Sheng and L. Chongguo, "Speech Service Applied in Local Region Based on RFID Technology", Bulletin of advanced technology research, vol. 3, no. 2, (2009).

[2] I. Betancourt, N. Pakravan and L. Shortbull, "Wireless Navigation Using RFID Network", EE198B Senior Project, unpublished.

[3] U. B. Ceipidor, G. Azzalin and M. Contenti, “A RFID System to Help Visually Impaired People Mobility, in press.

[4] M. Kourogi, N. Sakata, T. Okuma1 and T. Kurata, "Indoor/Outdoor Pedestrian Navigation with an Embedded GPS/RFID/Self-contained Sensor", 16th International Conference on Artificial Reality and Telexistence (ICAT2006), (2006), pp. 1310-1321.

[5] H. D. Chon, "Using RFID for Accurate Positioning, Presented at GNSS 2004", The 2004 International Symposium on GNSS/GPS Sydney, Australia, (2004).

[6] Y. Kobayashi, T. Takashima, T. Hayashi and H. Fujimoto, "Gait analysis of people walking ontactile ground surface indicators", in IEEE Transaction on Rehabilitation Engineering, 2005, vol. 13, no. 1, (2005), pp. 53-59.

[7] W. Lirong and Z. Ji, "Research of navigation and positioning at local area based on RFID", 2010" International Conference on Computer Application and System Modeling (ICCASM 2010), (2010).

[8] B. Duanyuan, "Research of region navigation based on Radio Frequency Identification", 2010 International Conference on Computer Mechatronics Control and Electronic Engineering, (2010).

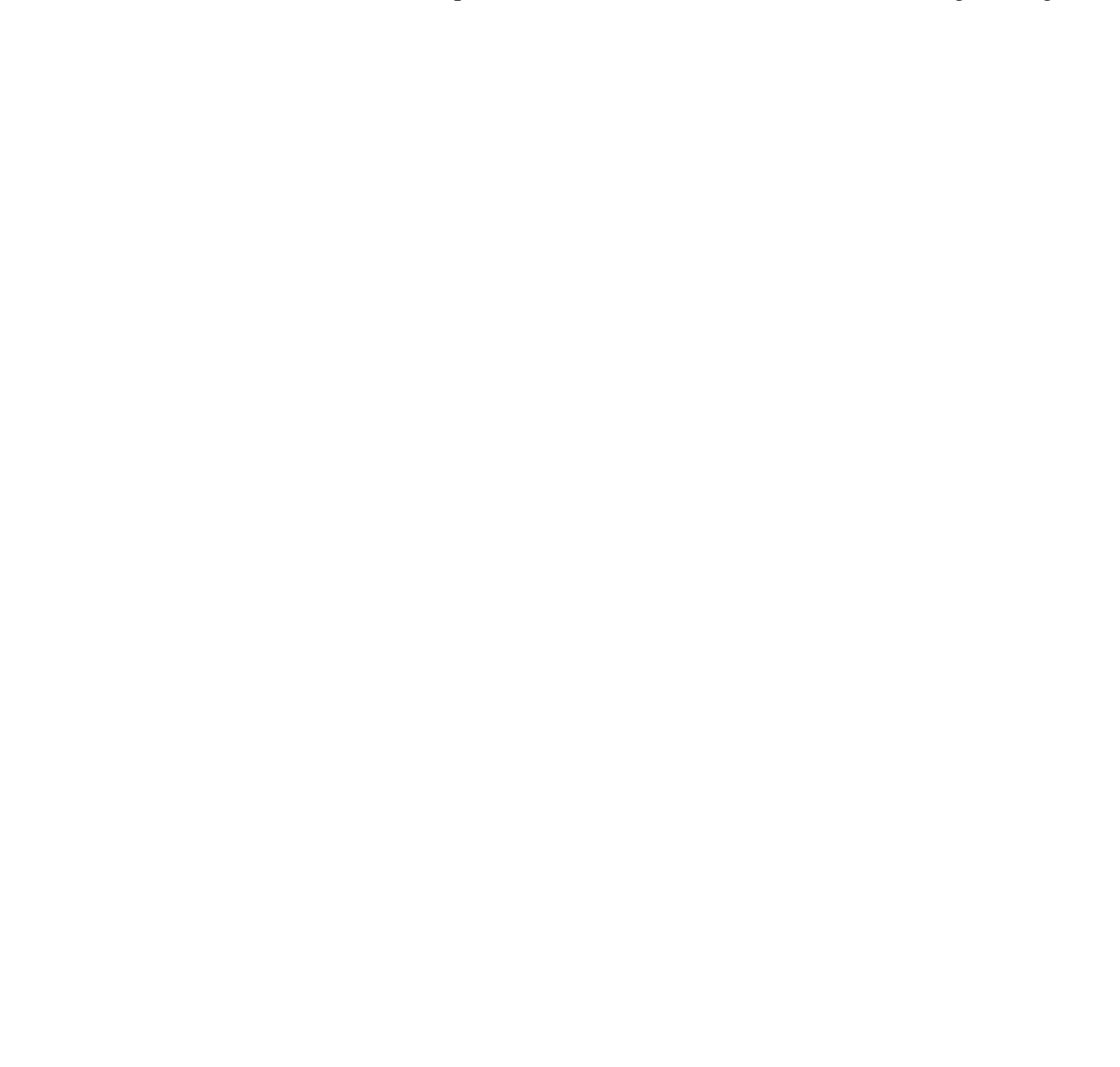

